\title{
"Cardiovascular Shower" Infective Endocarditis Causing Multiple Septic Emboli: A Case Report and a Review of Related Literature
}

\author{
Franz Michael M. Magnaye ${ }^{\mathrm{a}, \mathrm{c}}$, Marc Denver A. Tiongson ${ }^{\mathrm{b}}$, Rich Ericson C. King ${ }^{\mathrm{a}}$, \\ Roland Reuben B. Angeles ${ }^{\mathrm{a}}$, Mithi Kalayaan S. Zamora ${ }^{\mathrm{a}}$, Nashiba Daud ${ }^{\mathrm{b}}$, \\ Joseph Justin Regalado ${ }^{\mathrm{b}}$, Richard Henry P. Tiongco ${ }^{\mathrm{a}, \mathrm{b}}$
}

\begin{abstract}
Infective endocarditis (IE) is the infection of the endocardium or valves of the heart. Morbidity and mortality rates are high if not recognized early and left untreated. Complications such as heart failure, embolism and aneurysmal formation further increase mortality risk. We present a complicated case of IE where these complications coexisted in a single patient. A 27-year-old male diagnosed with rheumatic heart disease (RHD) presented with 3-month history of febrile episodes and 1-week history of left-sided weakness and facial asymmetry. Physical examination revealed subconjunctival hemorrhages, left-sided hemiparesis and left central facial palsy. On auscultation, he had murmurs suggestive of mitral regurgitation and stenosis, and aortic regurgitation. Two-dimensional echocardiography revealed mitral and aortic regurgitation with multiple large-sized vegetations at both valves. Further workup showed a right middle cerebral artery (MCA) infarct, right MCA mycotic aneurysms, and bilateral kidney infarcts with secondary infectious glomerulonephritis and splenic infarct. Blood cultures were negative. He was managed as culture-negative definite IE and was started on ceftriaxone and gentamycin which was later shifted to vancomycin due to lack of clinical improvement. During the course, he also developed severe abdominal pain - CT angiography of the abdomen revealed acute mesenteric ischemia of the superior mesenteric artery, which was managed conservatively. Double valve replacement was contemplated, but acute respiratory failure from hospital-acquired pneumonia posed higher surgical risks. Unfortunately, the patient eventually succumbed to brain herniation from new-onset multiple cerebral infarcts. Literature suggests rarity of multiple complications of IE occurring in a single patient. Because high mortality associated with complications can be averted by early recognition and early intervention, multiple embolisms should be
\end{abstract}

Manuscript submitted June 6, 2018, accepted June 20, 2018

aDepartment of Medicine, University of the Philippines-Philippine General Hospital, Manila, Philippines

${ }^{b}$ Section of Cardiology, University of the Philippines-Philippine General Hospital, Manila, Philippines

${ }^{\mathrm{c} C}$ Corresponding Author: Franz Michael M. Magnaye, Department of Medicine, University of the Philippines -Philippine General Hospital, Manila, Philippines. Email: franzmagnaye@yahoo.com

doi: https://doi.org/10.14740/jmc3102w suspected in a patient who already presented embolism in one organsystem.

Keywords: Rheumatic heart disease; Aneurysm infected; Mesenteric ischemia; Splenic infarction; Embolism; Endocarditis; Bacterial; Endocarditis

\section{Introduction}

Infective endocarditis (IE) is a deadly disease associated with high mortality and severe complication [1]. The current inhospital mortality rate for patients with IE is $15-20 \%$ with 1 -year mortality approaching $40 \%$ [2]. Likewise, IE is associated with high morbidity rates. As mentioned in various studies, complications may appear in $60 \%$ of patients with IE [3-6].

According to International Collaboration on EndocarditisProspective Cohort Study (ICE-PCS), the most common complication of IE is congestive heart failure at $32 \%$, followed by non-stroke embolization (renal, splenic or mesenteric infarcts) at $23 \%$ and stroke which occurs in $17 \%$ [2]. Moreover, rare complications such as presence of mycotic aneurysms occur in $2-4 \%$ of cases only [3-6]. No current data are available at present on how these complications may co-exist in a single patient, making management quite challenging to physicians who encounter such patients.

This case report demonstrates a case of IE that unfortunately had multiple complications. The rarity lies in the existence of these common and rare complications in a single patient, all of which unfortunately contributed to the patient's demise.

\section{Case Report}

A 27-year-old male, diagnosed case of rheumatic heart disease (RHD) since childhood, came in the emergency room with a 3-month history of persistent low-grade febrile episodes and unintentional weightloss. One week prior to admission, he had sudden-onset left-sided weakness and facial asymmetry. He had no other comorbidities and denied having any vices or any 


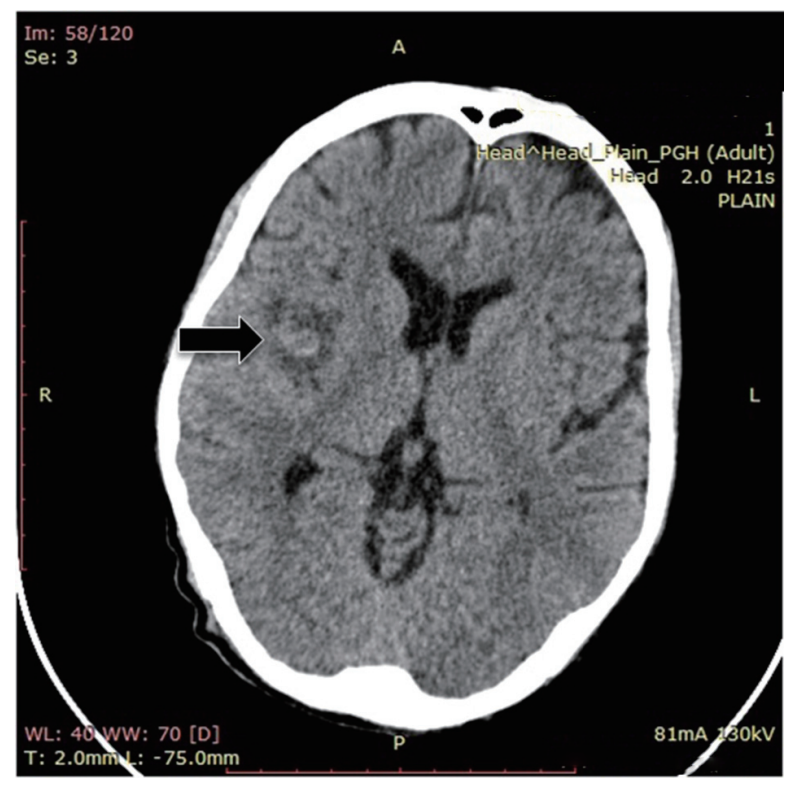

Figure 1. Plain cranial CT scan showing right middle cerebral infarct with hemorrhagic conversion.

\section{history of intravenous drug use.}

He was seen at the Emergency Department in hypotension requiring vasopressor support. Pertinent physical examination revealed pin-point hemorrhage on the left conjunctiva and petechial rashes on the chest. He had dynamic precordium and the following murmurs on auscultation: grade $4 / 6$ systolic murmur and grade 3/6 diastolic murmur at the apex

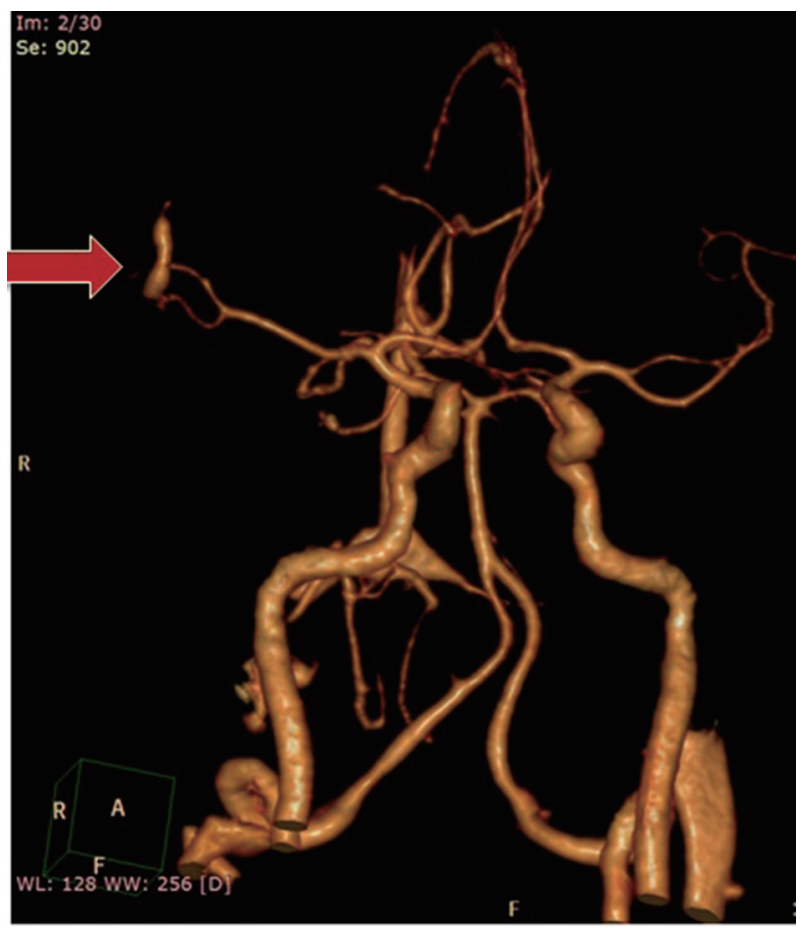

Figure 2. Cranial CT angiography showing an aneurysm at M2 segment of the right middle cerebral artery (red arrow).
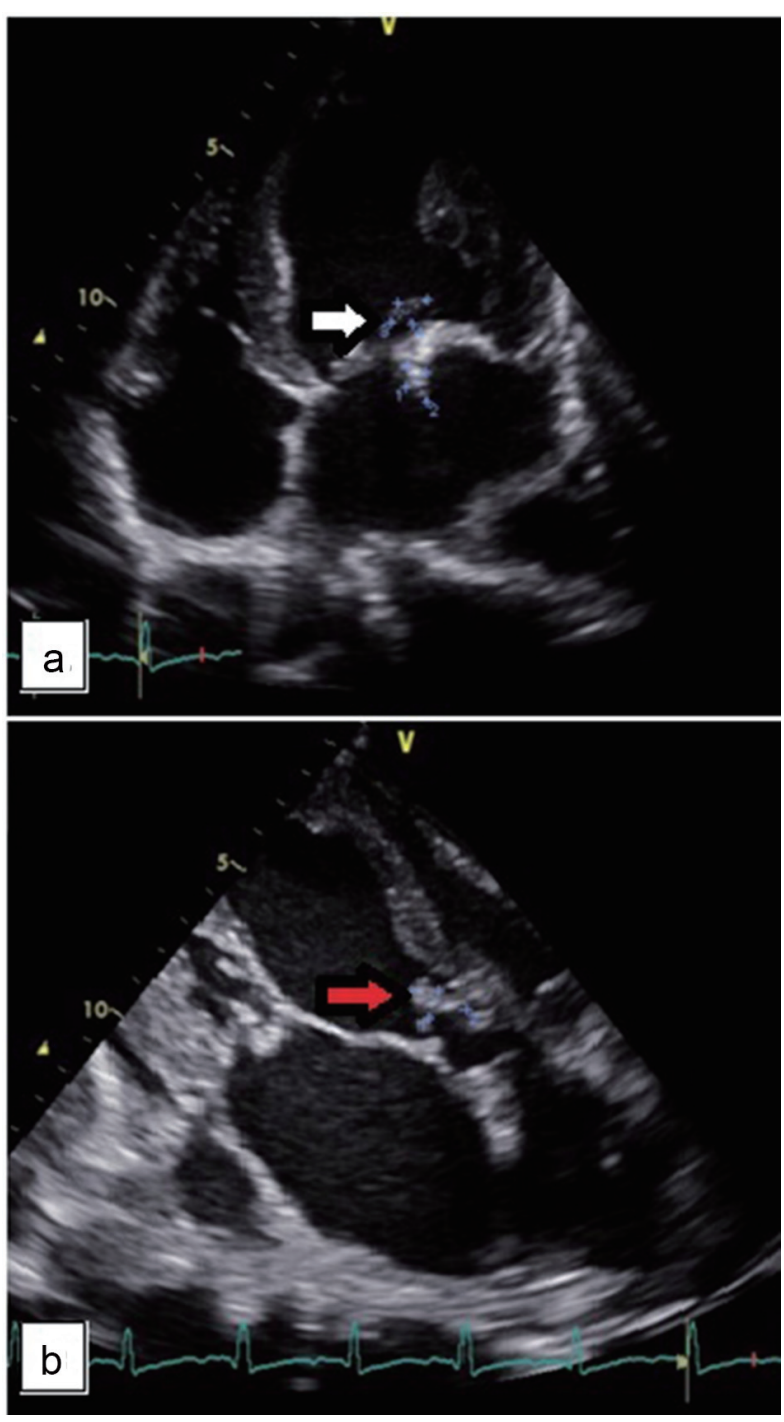

Figure 3. (a) An echogenic density attached to the mitral valve measuring $5 \times 12 \mathrm{~mm}$. (b) An echogenic density attached to the aortic valve measuring $16 \times 8 \mathrm{~mm}$.

and grade $3 / 6$ murmur at the second intercostal space (ICS) right parasternal border. His abdomen was soft and non-tender; no osler nodes, janeway lesions or splinter hemorrhages were noted. Neurological examination showed left central facial palsy and left hemiparesis. Stat cranial CT scan revealed a small infarct at the right middle cerebral artery with hemorrhagic conversion (Fig. 1). Initial laboratories revealed: anemia (Hgb 94g/L), leukocytosis (white blood cell (WBC): 21.3 $\times 10^{9} / \mathrm{L}$ with neutrophilic predominance), elevated creatinine at $181 \mu \mathrm{mol} / \mathrm{L}$, microscopic hematuria (red blood cell (RBC): $449 / \mu \mathrm{L}$ with $81 \%$ dysmorphic $\mathrm{RBC})$, pyuria $(22 / \mu \mathrm{L})$ and bacteriuria $(321 / \mu \mathrm{L})$. Focused echocardiography done revealed a dilated left ventricle with good wall motion and contractility, estimated ejection fraction of $58 \%$, mitral stenosis with moderate mitral regurgitation with multiple vegetations at the anterior and posterior mitral leaflet and subchondral apparatus, aortic valve sclerosis with severe aortic regurgitation with veg- 


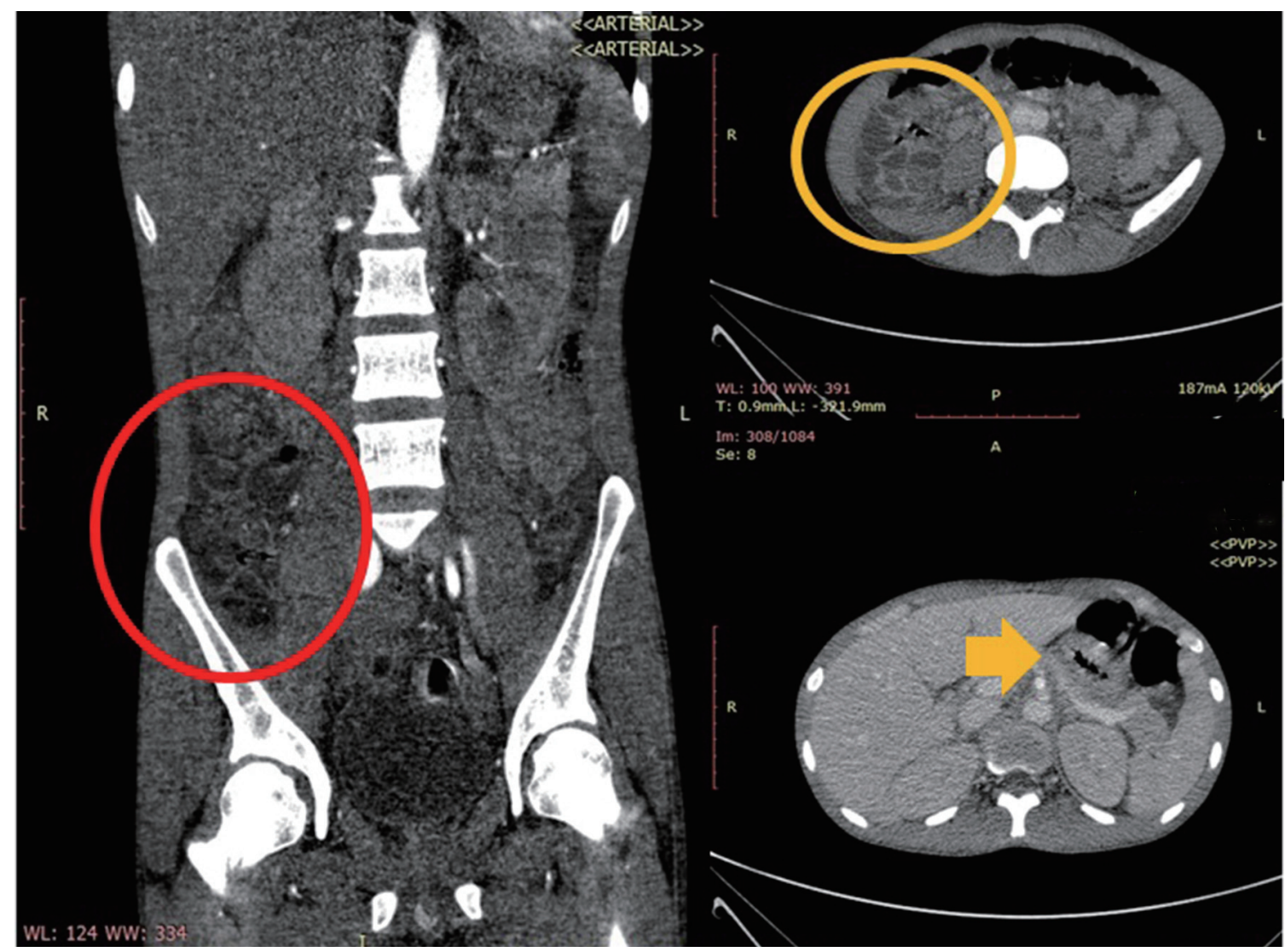

Figure 4. Abdominal CT angiography showing bowel wall thickening involving the stomach (yellow arrow), ascending colon (coronal cut: red circle; axial cut: yellow circle) which follows the superior mesenteric artery distribution.

etation. Three sets of blood cultures revealed no growth. IE was diagnosed on the basis of one major criteria (echocardiographic finding) and four minor criteria: 1) fever; 2) vascular phenomenon - embolic stroke, subconjunctival hemorrhage; 3 ) predisposing heart condition (RHD); and 4) immunologic from possible infectious glomerulonephritis. He was started on ceftriaxone and gentamicin.

We suspected possible mycotic aneurysm hence cranial CT angiography was done which revealed a fusiform aneurysmal dilatation of the superior branch (anterior sulcal artery) of the M2 segment of the right middle cerebral artery with an involved length of approximately $6-9 \mathrm{~mm}$ with its maximum width of $4 \mathrm{~mm}$. At the inferior aspect of the abovementioned fusiform dilatation arises a posteriorly directed narrow necked saccular aneurysm, its base measuring $2.1 \mathrm{~mm}$ with approximate length of $8.7 \mathrm{~mm}$ and a maximum body width of $3.8 \mathrm{~mm}$ (Fig. 2). Neurosurgery service suggested continuation of IV antiobiotic therapy and monitoring of resolution of the aneurysm after 4 - 6 weeks.

Febrile episodes persisted despite negative surveillance blood cultures. Antibiotics were escalated to vancomycin and ceftriaxone. Official two-dimensional echo results revealed a mobile echogenic density attached to the mitral valve $5 \times 12$ mm (in PLAX view) and $6 \times 9 \mathrm{~mm}$ (in SAX view). There is also a mobile echogenic density attached to the aortic valve measuring $16 \times 8 \mathrm{~mm}$ (in PLAX view) and $9 \times 6 \mathrm{~mm}$ (in SAX view) (Fig. 3). The patient was referred to Thoracic and Cardiovascular Surgery Division. He was advised urgent mitral and aortic valve replacement.

On day 21 of admission, patient suddenly experienced severe epigastric pain, with direct tenderness on the epigastric area, no rebound tenderness and no muscle guarding. Liver enzymes were slightly elevated, serum lipase was normal, alkaline phosphatase was slightly elevated and bilirubins were normal. Acute mesenteric ischemia (AMI) was considered. Urgent CT angiography of the abdomen was done revealing ascites, irregular rim enhancing wall thickening noted involving the stomach, ascending and transverse colon (superior mesenteric artery distribution) (Fig. 4). There was note of fairly defined, non-enhancing, wedge-shaped hypodense focus noted at the superior aspect of the spleen. Small fairly defined wedgeshaped hypodensities are also noted in the superior and inferior aspects of the left kidney and inferior aspect of the right kidney. Findings were consistent with AMI, splenic infarct and renal infarcts. Surgery Department suggested initiation of anticoagulants and vasodilators and for close monitoring as initial management for AMI. Adequate pain control was ensured.

On day 23 of admission, patient had acute respiratory failure due to acquired hospital acquired pneumonia. Sputum studies later revealed Acinetobacter baumanii, MDRO, sensitive only to colistin and minocycline. Patient's antibiotics were shifted to meropenem and colistin, while vancomycin was 
continued. Patient was transferred to the intensive care unit for closer monitoring. There is still an urgent indication to do the cardiac surgery; however, due to the critical condition of the patient, the team decided to continue optimizing the patient's clinical status, and treat the present pneumonia prior to valve replacement.

Surveillance cranial CT angiography after 5 weeks of antibiotics revealed disappearance of the previously noted fusiform aneurysmal dilatation of the superior branch (anterior sulcal artery) of the M2 segment of the right middle cerebral artery. There was a decrease in the sized of the previously noted narrow neck saccular aneurysm. A follow-up echocardiography was done revealing interval decrease in the size of the vegetations. There was progression of the valvular lesions; however, severe mitral valve regurgitation and severe aortic valve regurgitation are both indication for urgent surgery, but the patient was still deemed high risk due to comorbidities.

Later in the course, the patient developed progressive decrease in sensorium. Repeat cranial CT scan revealed newonset multiple infarcts. Medical decompression with mannitol was done; however, patient eventually succumbs due to brain herniation.

\section{Discussion}

The case features IE with multiple complications and it cannot be overemphasized that early recognition of IE together with its familiar and rare complications is associated with more favorable outcomes. Diagnosis of IE must be made at once in order to initiate therapy, and a delay in diagnosis may lead to severe complications. IE presents in various clinical pictures and it is in the physician's good judgment to have a high clinical suspicion to look for IE complications, no matter it is common or rare. For this case, we noted that intracranial mycotic aneurysm presenting as acute ischemic stroke, acute mesenteric ischemia presenting as severe abdominal pain and asymptomatic renal and splenic infarcts, all coexisted in a single patient.

Early surgical intervention could have altered the outcome of the case. Multiple studies have found out that heart surgery was noted to be an independent risk predictor for decreased mortality [2, 5, 7-9]. Current threshold for early surgical treatment has lowered according to the cohort study by ICE-PCS, revealing that $48 \%$ of patients were subjected to early surgical intervention [2]. Cardiac surgery has a positive influence on the mortality rate (odds ratio: 0.475 ) stating that those who underwent cardiac surgery during the same hospitalization, had a better outcome than other patients [10]. According to ESC Guidelines for Infective Endocarditis 2015, reasons to consider early surgery in the active phase (i.e. while the patient is still receiving antibiotic treatment) are to avoid progressive HF and irreversible structural damage caused by severe infection and to prevent systemic embolism [11], both occurring in our patient. An interesting study by Kang et al, a randomized controlled trial (RCT) comparing early surgery versus conservative management of IE, revealed that majority of those who had conventional therapy eventually needed the surgery during the same hospitalization due to development of urgent indication to surgery and presence of symptoms or left ventricular dysfunction more than 2 weeks [12]. Surgery is deemed warranted for patients with high-risk features that make the possibility of cure with antibiotic treatment unlikely and who do not have comorbid conditions or complications that make the prospect of recovery remote [11].

Literature suggests benefit of early surgical intervention on IE patients; however, certain subsets of patient characteristics are associated with high mortality rates. For this case, the surgery was deemed high risk during the later part of the course due to the following reason: presence of double valve IE necessitating a more extensive procedure, presence of worsening heart failure, concomitant severe pulmonary infection and other contributory factors such as the acute mesenteric ischemia, acute kidney injury and recent cerebrovascular disease (CVD) infarct. Regarding double valve surgery, a study by Sheikh et al revealed that it was in itself associated with a high risk of operative deaths (death within 90 days) [13]. In their study, $16 \%$ of patients who underwent double valve surgery died. Furthermore, the same study have shown that those with preoperative stroke had a $23.1 \%$ mortality rate and those who had urgent and emergent timing of surgery had $18.4 \%$ risk of mortality [13]. Despite reported good outcomes of early surgery, there are certain subsets of patients who are deemed high risk for perioperative mortality and therefore, decisions in management still need to be individualized. According to the ICE-PCS, these features are associated with high in-house mortality rate: age, prosthetic valve endocarditis, elevated erythrocyte sedimentation rate (ESR), radiographic pulmonary edema, Staphylococcus aureus IE, coagulase negative staphylococcus-IE, viridans strep-IE, mitral valve vegetation, paravalvular complications and surgery during this period [2].

This patient is at a high risk for embolic events according to various studies. Those who are high risk for embolic events include the presence of vegetations on the mitral valve and vegetations larger than $10 \mathrm{~mm}$ detected [2]. This is noted in the study of Thuny et al, which showed that the rate of symptomatic cerebral embolisms (transient ischemic attack (TIA) and ischemic strokes) occurring after the beginning of antibiotic therapy (and after the first echo) was significantly higher in patients with vegetation length greater than $10 \mathrm{~mm}$ than in those with vegetation length less than $10 \mathrm{~mm}(8 \%(20 / 247)$ versus 3.2\% (8/249), $\mathrm{P}<0.03)$ [14]. Mitral valve lesions have been associated with higher rates of embolic complications compared to aortic valve vegetations $(25 \%$ and $10 \%$, respectively) [7]. Our patient is at very high risk for embolic events as he had double valve involvement, both with more than 10 mm-sized vegetations, had a previous embolic event and hence would have benefited with early surgical intervention.

For patients with neurological events, indication for cardiac surgery often remains and often strengthened as to prevent future embolic events, but must be balanced with perioperative risk and postoperative prognosis [11]. According to Thuny et al, the risk of postoperative neurological deterioration is low after a silent cerebral emboli or transient ischemic attack, and surgery is recommended without delay if an indication remains. After an ischemic stroke, cardiac surgery is not contraindicated unless the neurological prognosis is judged to be 
poor [14]. For patients with hemorrhagic strokes, however, it is recommended to postpone the surgery at least 1 month as it is associated with poor neurological prognosis [11].

Initiation of early antibiotics is crucial in the management of IE. Initiation of antibiotics decreases the risks of embolic events to $6-21 \%$ [15]. The addition of antiplatelet therapy did not reduce the risk of embolism based on a single study by Chan et al [16].

Patient presented with a rare complication of IE, cerebral mycotic aneuryms. This complication is rare, occurring in around $2-4 \%$ of cases, and the same as with the patient, more than half $(57.4 \%)$ are located in the distal branches of the medial cerebral artery [3-6]. Majority of mycotic aneurysms are asymptomatic, diagnosed only by imaging exams which emphasizes the need to suspect such in a patient with IE and neurological symptoms. Most unruptured mycotic aneurysms have an approximated mortality rate of $30 \%$ which resolves with antibiotic treatment and surveillance. No predictor of rupture has been identified and in contrast to non-infectious aneurysms, size does not appear to be a reliable predictor of potential rupture [11]. For this case, we noted significant decreased in the size of the mycotic aneurysm on IV antiobiotic therapy alone. According to Gillinov et al, there is no evidence that cardio-pulmonary bypass or heparin treatment predisposes mycotic aneurysms to rupture and the recommendation is not to clip or resect unruptured mycotic aneurysms before undertaking valve replacement [8].

AMI, regardless of etiology, is caused by a critical reduction in intestinal blood flow that frequently results in bowel necrosis and is associated with a high mortality [17]. Most common cause is still arterial occlusion, usually cardioembolic [18]. Medical management in general includes aggressive rehydration and the use of antibiotics, anticoagulation, vasodilators and inhibitors of reperfusion injury [17]. IE is quite an uncommon cause of AMI, in fact, rare according to a case report by Waqas et al, for which he was only able to find three other case reports of AMI from IE other than their case report [18]. Since it is not common, there are no well-established guidelines to handle such cases. The dilemma that we experience is whether there is a role in anticoagulation as a medical therapy versus surgery. Although role of anticoagulation in acute embolic mesenteric ischemia is well established [18], its role cannot be inferred the same for embolic ischemia from IE because the vegetation in IE is a mass of platelets, fibrin and microcolonies of microorganisms which may or may not be lyzed by anticoagulants. As of this writing, there were no hard recommendations that support the use of anticoagulant in the treatment of AMI from IE. Re-evaluating our management, we opted to proceed with medical management of AMI by giving vasodilators and anticoagulant and close observation was done. However, there is danger in the use anticoagulants in IE patients [18]. The European Society of Cardiology guidelines states that there is no indication for the initiation of antithrombotic drugs (thrombolytic drugs, anticoagulant or antiplatelet therapy) during the active phase of IE [11]. This is based on low levels of evidence where an increased risk of cerebral hemorrhage has been observed in patients with IE who were taking oral anticoagulants [18], especially those who had previous neurological event such as in our patient.
However, to our best opinion, there is still a role for medical management in AMI as there was no clinical deterioration of the AMI noted in our patient. Further studies are recommended in order to establish the role of medical management in AMI from IE; however, rarity of this complication served as limiting factor. Limited case reports such as this paper are the basis for management.

\section{Conclusions}

We have reported a case of IE causing multiple septic emboli. Early surgery could have decreased the risk of cardioembolic. We emphasize that immediate recognition and surgical interventions must be promptly done for patients with such conditions as complications arise due to delay in management. There is evidence of good outcome on patients subjected to early surgery as these patients are still good candidates for surgery; once other complications arise, preoperative risks and mortality rates increases.

\section{References}

1. Thuny F, Grisoli D, Collart F, Habib G, Raoult D. Management of infective endocarditis: challenges and perspectives. Lancet. 2012;379(9819):965-975.

2. Murdoch DR, Corey GR, Hoen B, Miro JM, Fowler VG, Jr., Bayer AS, Karchmer AW, et al. Clinical presentation, etiology, and outcome of infective endocarditis in the 21st century: the International Collaboration on Endocarditis-Prospective Cohort Study. Arch Intern Med. 2009;169(5):463-473.

3. Ducruet AF, Hickman ZL, Zacharia BE, Narula R, Grobelny BT, Gorski J, Connolly ES, Jr. Intracranial infectious aneurysms: a comprehensive review. Neurosurg Rev. 2010;33(1):37-46.

4. Pessinaba S, Kane A, Ndiaye MB, Mbaye A, Bodian M, Dia MM, Sarr SA, et al. Vascular complications of infective endocarditis. Med Mal Infect. 2012;42(5):213-217.

5. Fukuda W, Daitoku K, Minakawa M, Fukui K, Suzuki Y, Fukuda I. Infective endocarditis with cerebrovascular complications: timing of surgical intervention. Interact Cardiovasc Thorac Surg. 2012;14(1):26-30.

6. Yeates A, Mundy J, Griffin R, Marshall L, Wood A, Peters P, Shah P. Early and mid-term outcomes following surgical management of infective endocarditis with associated cerebral complications: a single centre experience. Heart Lung Circ. 2010;19(9):523-527.

7. Veiga VC, Carvalho JC, Amaya LE, Martins MS, Rojas SS. Meningitis as a complication of infective endocarditis. Rev Bras Ter Intensiva. 2012;24(3):308-311.

8. Gillinov AM, Shah RV, Curtis WE, Stuart RS, Cameron DE, Baumgartner WA, Greene PS. Valve replacement in patients with endocarditis and acute neurologic deficit. Ann Thorac Surg. 1996;61(4):1125-1129; discussion 1130.

9. Rossi M, Gallo A, De Silva RJ, Sayeed R. What is the optimal timing for surgery in infective endocarditis with 
cerebrovascular complications? Interact Cardiovasc Thorac Surg. 2012;14(1):72-80.

10. Male KR, Mathews A, Mower J. An unusual presentation of an unusual disease: infective endocarditis: a case report and review of the literature. Cases J. 2008;1(1):292.

11. Habib G, Lancellotti P, Antunes MJ, Bongiorni MG, Casalta JP, Del Zotti F, Dulgheru R, et al. 2015 ESC Guidelines for the management of infective endocarditis: The Task Force for the Management of Infective Endocarditis of the European Society of Cardiology (ESC). Endorsed by: European Association for Cardio-Thoracic Surgery (EACTS), the European Association of Nuclear Medicine (EANM). Eur Heart J. 2015;36(44):3075-3128.

12. Kang DH, Kim YJ, Kim SH, Sun BJ, Kim DH, Yun SC, Song JM, et al. Early surgery versus conventional treatment for infective endocarditis. N Engl J Med. 2012;366(26):2466-2473.

13. Sheikh AM, Elhenawy AM, Maganti M, Armstrong S, David TE, Feindel CM. Outcomes of double valve sur- gery for active infective endocarditis. J Thorac Cardiovasc Surg. 2009;138(1):69-75.

14. Thuny F, Avierinos JF, Tribouilloy C, Giorgi R, Casalta JP, Milandre L, Brahim A, et al. Impact of cerebrovascular complications on mortality and neurologic outcome during infective endocarditis: a prospective multicentre study. Eur Heart J. 2007;28(9):1155-1161.

15. Guler S, Sokmen A, Mese B, Bozoglan O. Infective endocarditis developing serious multiple complications. BMJ Case Rep. 2013;2013.

16. Chan KL, Dumesnil JG, Cujec B, Sanfilippo AJ, Jue J, Turek MA, Robinson TI, et al. A randomized trial of aspirin on the risk of embolic events in patients with infective endocarditis. J Am Coll Cardiol. 2003;42(5):775-780.

17. Berland T, Oldenburg WA. Acute mesenteric ischemia. Curr Gastroenterol Rep. 2008;10(3):341-346.

18. Waqas M, Waheed S, Haider Z, Shariff AH. Acute mesenteric ischaemia with infective endocarditis: is there a role for anticoagulation? BMJ Case Rep. 2013;2013. 